\title{
Exosomes secreted from miRNA-29b-modified mesenchymal stem cells repaired spinal cord injury in rats
}

\author{
Tao Yu(i) ${ }^{1,2 *}$, Cunju Zhao (i) $^{1,2 *}$, Shouzhi Hou ${ }^{3}{ }^{3}$, Weijie Zhou ${ }^{2}{ }^{2}$, Baoxin Wang (i) $^{2}$, and \\ Yunzhen Chen (ii) ${ }^{1}$ \\ ${ }^{1}$ Department of Spinal Surgery, Qilu Hospitial of ShanDong University, Jinan, Shandong, China \\ ${ }^{2}$ Department of Orthopedics, Liaocheng People's Hospitial, Liaocheng, Shandong, China \\ ${ }^{3}$ Department of Radiology, Liaocheng People's Hospitial, Liaocheng, Shandong, China
}

\begin{abstract}
Exosomes, a kind of extracellular vesicle, are promising therapeutic agents for spinal cord injury (SCl). This article aimed to investigate effects of exosomes secreted from miRNA-29b-modified bone marrow mesenchymal stem cells (BMSCs) on SCl. Exosomes were extracted from BMSCs transfected with miRNA-29b or negative control (miR NC). SCl rats were injected intravenously with exosomes (control exosomes, miRNA-29b exosomes) and BMSCs (miR NC, miRNA-29b) through the tail vein. The expression of miRNA-29b in spinal cord tissues of SCl rats was detected by qRT-PCR. The hind limb motor function was evaluated by Basso Beattie Bresnahan (BBB) score. The histopathological damage and neuronal regeneration in spinal cord tissues was observed by HE staining and immunohistochemistry, respectively. The injection of miRNA-29b exosomes and miRNA-29b BMSCs both significantly increased the expression of miRNA-29b in spinal cord tissues of $S C l$ rats $(P<0.05)$. Compared with $\mathrm{SCl}$ rats, rats in the miRNA-29b exosomes and the miRNA-29b groups exhibited improved SCl, including increased BBB score, NF200 and GAP-43 positive neurons, as well as decreased contractile nerve cell numbers and GFAP positive neurons (all $\mathrm{P}<0.05$ ). The relieving degree of $\mathrm{SCl}$ was significantly higher in the miRNA-29b exosomes group than in the miRNA-29b BMSCs group $(P<0.05)$. Exosomes secreted from miRNA-29b-modified BMSCs were effective in the repair of $\mathrm{SCl}$ in rats.
\end{abstract}

Key words: miRNA-29b; Exosomes; Spinal cord injury; BMSCs

\section{Introduction}

Spinal cord injury (SCl) is a serious central nervous system disease that leads to severe leg dysfunction and even lifelong paralysis (1). Primary mechanical $\mathrm{SCl}$ can quickly trigger secondary injury and induce inflammatory response and neuronal apoptosis in the injured area (2). Subsequently, astrocyte scars and syringomyelia are formed, resulting in the inhibition of axonal regeneration and dysfunction of motor and sensory function (3). The treatment of $\mathrm{SCl}$ is still difficult in clinical practice due to the poor regeneration ability of neurons.

In recent years, stem cell transplantation has become a hot spot in the treatment of SCl with the development of stem cell technology. The transplantation of bone marrow mesenchymal stem cells (BMSCs) has been considered an effective therapeutic strategy for $\mathrm{SCl}$, since it can inhibit inflammation and apoptosis, promote axonal regeneration and angiogenesis, reduce astrocyte scars and syringomyelia, and promote recovery of motor function (4). However, BMSCs transplantation also has some drawbacks, such as teratogenic tumorigenesis, immune rejection, pulmonary embolism, low transplantation rate, and survival rate $(5,6)$. Exosomes (diameter: $30-100 \mathrm{~nm}$ ) are extracellular vesicles released from cells into the extracellular space (7). As an important mediator of cell-cell interaction, exosomes can enter target cells through membrane receptors or endocytosis, and then participate in the regulation of biological information via transmitting mRNAs, non-coding small RNAs, proteins, etc. (8). Noteworthy, exosomes exert positive therapeutic effects on $\mathrm{SCl}$ through inhibiting neuronal apoptosis and inflammatory response (7). Huang et al. (9) found that the administration of mesenchymal stem cells (MSCs)-derived exosomes in $\mathrm{SCl}$ rats

Correspondence: Yunzhen Chen: <chenyunzhen23@163.com>

${ }^{*}$ Tao Yu and Cunju Zhao are co-first authors.

Received April 18, 2019 | Accepted October 14, 2019 
significantly attenuates the lesion size, as well as cellular apoptosis and inflammation in the injured spinal cord. Sun et al. (10) found that human umbilical cord MSCs-derived exosomes facilitate the healing of $\mathrm{SCl}$ through attenuating inflammation. Wang et al. (11) found that MSC-derived exosomes exert obvious neuroprotective effects on $\mathrm{SCl}$ by reducing $\mathrm{SCl}$-induced $\mathrm{A} 1$ astrocytes and inhibiting inflammation. Therefore, exosomes are expected to be an alternative to stem cells in the treatment of $\mathrm{SCl}$.

MicroRNAs (miRNAs) are a type of endogenous small RNAs with a length of 20-24 nucleotides (12). Some miRNAs contribute to the repair of damaged nerve tissues and cells, such as miR-340, miR-204, etc $(13,14)$. BMSCs can secrete exosomes with high levels of specific miRNA by pretransfection with specific miRNA plasmids (15). Previous studies have proven that miRNA-29 is involved in the repair of liver damage, myocardial ischemia-reperfusion injury, skeletal muscle injury, as well as human podocyte injury (16-19). However, the regulatory role of miRNA-29 on $\mathrm{SCl}$ is still unclear. In this study, the effects of exosomes secreted from miRNA-29b-modified BMSCs on SCI were evaluated. Our findings may provide important clinical theoretical basis and guidance for the treatment of $\mathrm{SCl}$.

\section{Material and Methods}

\section{Animals}

A total of 80 female Sprague-Dawley rats weighing 230-250 g were purchased from Shandong Lukang Pharmaceutical Co., Ltd. (China). Rats were routinely housed in specific pathogen-free animal rooms at $22-26^{\circ} \mathrm{C}$ with $40-60 \%$ humidity. All rats were given free access to water and feed. All animal experiments in this study met the requirements of the animal ethics committee of Qilu Hospitial of ShanDong University.

\section{Isolation and culture of BMSCs}

One-month-old (100-120 g) rats were anesthetized by intraperitoneal injection of $1 \%$ pentobarbital (dose, $80 \mathrm{mg} /$ $\mathrm{kg}$ ). After being disinfected with $75 \%$ alcohol, the femur and tibia of the rat were obtained by removing the surface muscles and fascia. Bone marrow was then obtained by rinsing with Hanks solution. After 5 min of centrifugation at $170 \mathrm{~g}\left(4^{\circ} \mathrm{C}\right)$, BMSCs at the bottom of the centrifuge tube were remixed evenly with Hanks solution. Followed by 5 min of centrifugation at $1050 \mathrm{~g}\left(4^{\circ} \mathrm{C}\right)$, BMSCs were suspended in LG-DMEM cell culture medium containing $10 \%$ fetal bovine serum (FBS). Then, BMSCs were seeded in a sterile culture flask in an incubator at $37^{\circ} \mathrm{C}$ with $5 \% \mathrm{CO}_{2}$. The medium was replaced with fresh medium every 2 days. After three generations, BMSCs at logarithmic growth phase were used for the following assays.

\section{Identification of surface markers in BMSCs}

BMSCs were added into 1.5-mL centrifuge tubes at a density of $1 \times 10^{5}$ cells per tube. After $5 \mathrm{~min}$ of centrifugation at $170 \mathrm{~g}\left(25^{\circ} \mathrm{C}\right)$, the supernatant was discarded and BMSCs at the bottom of tubes were resuspended in $100 \mu \mathrm{L}$ D-Hanks solution. Then, FITC-labeled anti-rat CD44, PE-labeled anti-rat CD73, FIC-labeled antimouse IgG, and PE-labeled anti-mouse IgG antibodies were added to the tubes. D-Hanks solution was added to one of the tubes for control. All tubes were incubated at room temperature for $\mathbf{4 5} \mathrm{min}$ in the dark. After washing 3 times with D-Hanks, BMSCs in each tube were analyzed by flow cytometry.

\section{BMSCs transfection and exosomes extraction}

BMSCs were inoculated on 24-well plates at a density of $2 \times 10^{6}$ cells/well. After $48 \mathrm{~h}$ of culturing, BMSCs were transfected with miR-29b recombinant lentiviral vector (miR-29b-LV) and recombinant LV carrying enhanced green fluorescent protein (EGFP) reporter gene (NCEGFP-LV) (Ruibo Biotechnology Co., Ltd., China). These transfected BMSCs were named as miRNA-29b group and miR NC group, respectively. The multiplicity of infection was 20. Three days after the transfection, BMSCs were stained with DAPI for $1 \mathrm{~h}$. The EGFP fluorescence was observed under a fluorescence microscope. BMSCs were collected for subsequent assays when the transfection efficiency was more than $95 \%$. In addition, exosomes were extracted from the supernatant of each well using the Exo Quick-TC kit (SBI, USA) in strict accordance with the instructions.

\section{Identification of exosome protein markers by western blot}

Exosomes were quantified by bicinchoninic acid (BCA) and subjected to sodium dodecyl sulfate polyacrylamide gel electrophoresis (SDS-PAGE). Then, the proteins were transferred onto a polyvinylidene fluoride (PVDF) membrane, blocked with 5\% skim milk, and incubated with primary antibodies (rabbit anti-rat CD9 monoclonal antibody, ab92726; mouse anti-rat CD63 monoclonal antibody, ab108950; rabbit anti-rat CD81 monoclonal antibody, ab109201; rabbit anti-rat GAPDH polyclonal antibody, ab9485; $1: 1000$, Abcam, UK) for $12 \mathrm{~h}$ at $4^{\circ} \mathrm{C}$. After washing 3 times with Tris-buffered saline and Tween 20 (TBST), the membrane was incubated with horseradish peroxidase-labeled secondary antibodies (goat anti-mouse IgG, goat anti-rabbit IgG, 1:5000, Beijing Zhongshang Jinqiao Biotechnology Co., Ltd., China) for $2 \mathrm{~h}$ at room temperature. After washing three times with TBST, the protein bands were detected using a Kodak film developer (Fujifilm, Japan).

\section{Construction of $\mathrm{SCl}$ model in rats}

Intraperitoneal injection of $1 \%$ pentobarbital sodium at a dose of $50 \mathrm{mg} / \mathrm{kg}$ was used to anesthetize rats. Rats were placed on the operating table and disinfected. A 2$3 \mathrm{~cm}$ incision was made at the midline of the back using the T10 spinous process as the center. The vertebrae 
were exposed after the surface muscles were separated. Spinous processes and lamina were removed to fully expose the T10 spinal cord. During this procedure, the dura mater was completely preserved. Using a standard striking device, the T10 spinal cord was hit with a striking force of $2 \mathrm{~N}$. The wound was stitched after being washed with penicillin saline. The successfully established rat model of $\mathrm{SCl}$ met the following characteristics, including congestive edema of T10 segment, twitch of tail and hind limb, and Basso Beattie Bresnahan (BBB) score less than 10.

\section{Grouping and intervention}

A total of $100 \mathrm{SCl}$ rats were randomly divided into 5 groups: SCl, control exosomes, miRNA-29b exosomes, miR NC, and miRNA-29b ( $n=20$ per group). One hour after modeling, rats were injected intravenously with $500 \mu \mathrm{L}$ control exosomes (secreted from miR NC BMSCs) or miRNA-29b exosomes (secreted from miRNA-29b BMSCs) at a concentration of $200 \mu \mathrm{g} / \mathrm{mL}$ through the tail vein. Rats injected intravenously with miR NC BMSCs and miRNA-29b BMSCs $\left(10^{7}\right.$ cells $\left./ \mathrm{mL}, 10 \mu \mathrm{L}\right)$ were enrolled as miR NC group and miRNA-29b group, respectively. Rats injected intravenously with physiological saline were enrolled as $\mathrm{SCl}$ group. After the injection, penicillin was injected at a dose of $2 \times 10^{5} \mathrm{U} / \mathrm{kg}$ once a day for 3 days, and urination care was given 3 times a day until resuming active urination.

\section{Motor function assessment}

The hind limb motor function of rats was evaluated by BBB score at 3 days, 1, 2, 4, and 8 weeks post-injection as previously described (20).

\section{Spinal cord tissue collection}

At 3 days, 1, 2, 4, and 8 weeks post-injection, 4 rats in each group were randomly selected and deeply anesthetized. The thoracic cavity of rats was opened to expose the heart. A total of $250 \mathrm{~mL}$ physiological saline was perfused into the ascending aorta to adequately flush the blood in tissues. Then, 4\% paraformaldehyde was perfused until the liver was whitened and stiffened. The central segment of spinal cord tissues at the injury site (T9-10, about $1 \mathrm{~cm}$ in length) was removed and stored in liquid nitrogen.

\section{Detection of miRNA-29b expression by qRT-PCR}

Total RNA was extracted from spinal cord tissues 1 week post-injection using Trizol reagent according to the manufacturer's instructions. RNA samples were reversely transcribed using cDNA Reverse Transcription Kit (Applied Biosystems, USA). qRT-PCR was carried out by the Fast Start Universal SYBR Green Mastermix (Roche, USA). All primers were synthesized by Shanghai Sangon Biotech Co., Ltd., China. Primer sequences were: miRNA29b, forward: 5'-CTGACGGAGTTCCTCCAGTTC-3', reverse: 5'-GAGGTTCCCCGAGAAGACGAT-3' and U6, forward: 5'-CTCGCTTCGGCAGCACA-3', reverse: 5'-AC GCTTCACGAATTTGCGT-3'. Data were processed by the $2^{-\Delta \Delta C T}$ method, and relative miRNA-29b expression was normalized to U6.

\section{Hematoxylin and eosin (HE) staining}

Four weeks after injection, spinal cord tissues (about $0.5 \mathrm{~cm}$ around the injured site) were fixed with $4 \%$ paraformaldehyde for $24 \mathrm{~h}$, embedded in paraffin, and sliced at a thickness of $6 \mu \mathrm{m}$. These tissue sections were subjected to xylene dewaxing, alcohol gradient dehydration and hydration, followed by $\mathrm{HE}$ staining. After being dehydrated with alcohol and xylene, sections were sealed with neutral resin and observed under an optical microscope (Olympus, Japan).

\section{Immunohistochemistry}

Spinal cord sections were subjected to xylene dewaxing, gradient alcohol rehydration, and antigen retrieval with boiling $0.01 \mathrm{M}$ citrate buffer. Endogenous peroxidase activity was eliminated by 15 min of incubation with $3 \%$ hydrogen peroxide. Then, these sections were incubated with goat serum blocking solution for $15 \mathrm{~min}$ at room temperature and incubated with primary antibodies (rabbit anti-rat NF200, ab8135, GFAP, ab7260, GAP-43, ab75810, $1: 100$, Abcam, UK) for $12 \mathrm{~h}$ at $4^{\circ} \mathrm{C}$. After washing 3 times with PBS, sections were incubated with horseradish peroxidase-labeled goat anti-rabbit IgG (1:500, Beijing Zhongshang Jinqiao Biotechnology Co., Ltd.) for $15 \mathrm{~min}$ at $37^{\circ} \mathrm{C}$. Followed by DAB color reaction and hematoxylin counterstaining, sections were sealed with neutral gum and observed under an optical microscope (Olympus). Positive cells were counted in 5 random fields of view.

\section{Statistical analysis}

All experiments were repeated 3 times independently. Data were analyzed by SPSS 21.0 statistical software (USA). All quantitative data are reported as means \pm SD. Comparison between different groups was determined by one-way ANOVA followed by post hoc test (two groups). $\mathrm{P}<0.05$ was considered statistically significant.

\section{Results}

\section{Identification of BMSCs}

After 10 days of primary culture, a large number of fusiform BMSCs were observed (Figure 1A). After three generations, the morphology of BMSCs was consistent with the morphological characteristics of BMSCs (21). Flow cytometry showed that more than $90 \%$ of BMSCs showed positive expression of CD44, CD73, and CD90 (Figure 1B). After $72 \mathrm{~h}$ of transfection with miRNA-29b and miR NC, more than $95 \%$ of BMSCs showed positive expression of EGFP (Figure 1C and D). Then, exosomes were extracted from transfected BMSCs. Western blot showed that miRNA-29b exosomes and control exosomes 
A



\section{C}

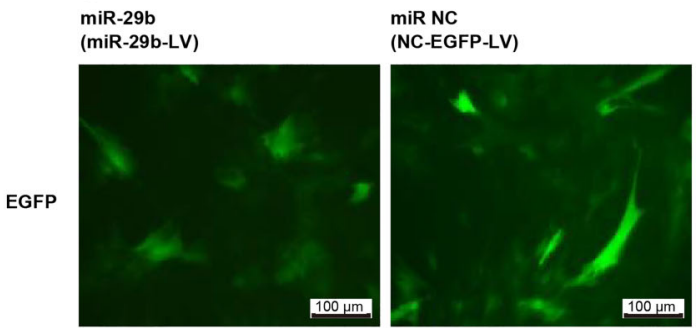

B
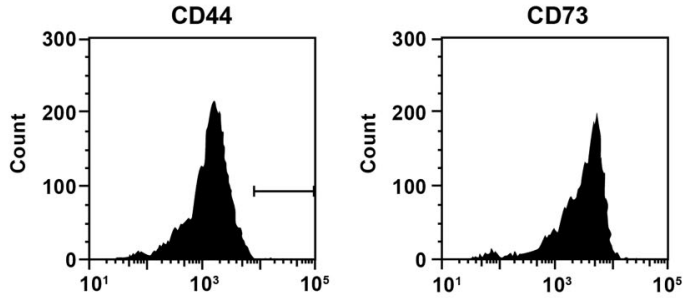

D





E



Figure 1. Identification of bone marrow mesenchymal stem cells (BMSCs) and exosomes. A, Fusiform BMSCs were observed under a microscope after primary culture for 10 days (bar=50 $\mu \mathrm{m}$ ). B, Positive expression of CD44, CD73, and CD90 was observed in BMSCs by flow cytometry. C, Enhanced green fluorescent protein (EGFP) was observed in BMSCs transfected with miR-29b-LV or NC-EGFP-LV for $72 \mathrm{~h}($ bar $=100 \mu \mathrm{m})$. D, Virus transfection efficiency was more than $95 \%$ in BMSCs transfected with miR-29b-LV or NC-EGFP-LV for 72 h. E, Positive expression of CD63, CD81, and CD9 was observed in exosomes secreted from transfected BMSCs by western blot. Data are reported as means \pm SD.

showed positive expression of CD63, CD81, and CD9 (Figure 1E).

\section{miRNA-29b expression in spinal cord tissues of $\mathrm{SCl}$ rats}

One week after injection, miRNA-29b expression in spinal cord tissues of $\mathrm{SCl}$ rats in each group was detected by qRT-PCR. As shown in Figure 2, the relative expression of miRNA-29b was significantly higher in the miRNA$29 \mathrm{~b}$ exosomes group than in the control exosomes group $(P<0.05)$. The relative expression of miRNA-29b was also significantly increased in the miRNA-29b group compared with the miR NC group $(P<0.05)$. No significant difference for miRNA-29b expression was observed between the $\mathrm{SCl}$ and control exosomes and miR NC groups (Figure 2).

\section{Injection of miRNA-29b exosomes accelerated the motor function of $\mathrm{SCl}$ rats}

Reduced feeding and mobility were found in $\mathrm{SCl}$ rats after surgery. Severe hind-limb motor dysfunction and loss of spontaneous urinary function were also observed in $\mathrm{SCl}$ rats. All rats received urination care (3 times/day), and no urinary retention-induced bladder rupture occurred. The BBB scores in rats injected with BMSCs and exosomes increased with time in a time-dependent manner. The BBB scores were significantly higher in the miRNA-29b exosomes group than in the control exosomes group at 2,4 , and 8 weeks post-injection $(P<0.05)$, and were also significantly higher in the miRNA-29b group than in the miR NC group at 1, 2, 4, and 8 weeks postinjection $(P<0.05)$. Compared with the miRNA-29b group, the miRNA-29b exosomes group exhibited significantly higher BBB scores at 1, 2, 4, and 8 weeks post-injection $(P<0.05)$ (Figure 3).

\section{Injection of miRNA-29b exosomes alleviated histopathological damage in spinal cord tissues of $\mathrm{SCl}$ rats}

Severe structural disorder, rupture of the vessel wall, and neuronal edema were observed in spinal cord tissues of $\mathrm{SCl}$ rats. The boundary between the white matter and the gray matter was blurred, and enhanced neuronal nuclear staining was also observed in the spinal cord gray matter. At 4 weeks post-injection, the number of contractile nerve cells in the SCl group was significantly decreased by the injection of BMSCs or exosomes $(P<0.05)$. In addition, the number of contractile nerve cells in the miRNA-29b exosomes group was significantly lower than in the control 




Figure 2. MiRNA-29b expression in spinal cord tissues of spinal cord injury $(\mathrm{SCl})$ rats. Data are reported as means $\pm \mathrm{SD}$. ${ }^{*} \mathrm{P}<0.05$ vs $\mathrm{SCl}$ group; ${ }^{\&} \mathrm{P}<0.05$ vs control exosomes; ${ }^{\#} \mathrm{P}<0.05$ vs miR $\mathrm{NC}$; ${ }^{\mathrm{P}}<0.05$ vs miRNA-29b exosomes (ANOVA).

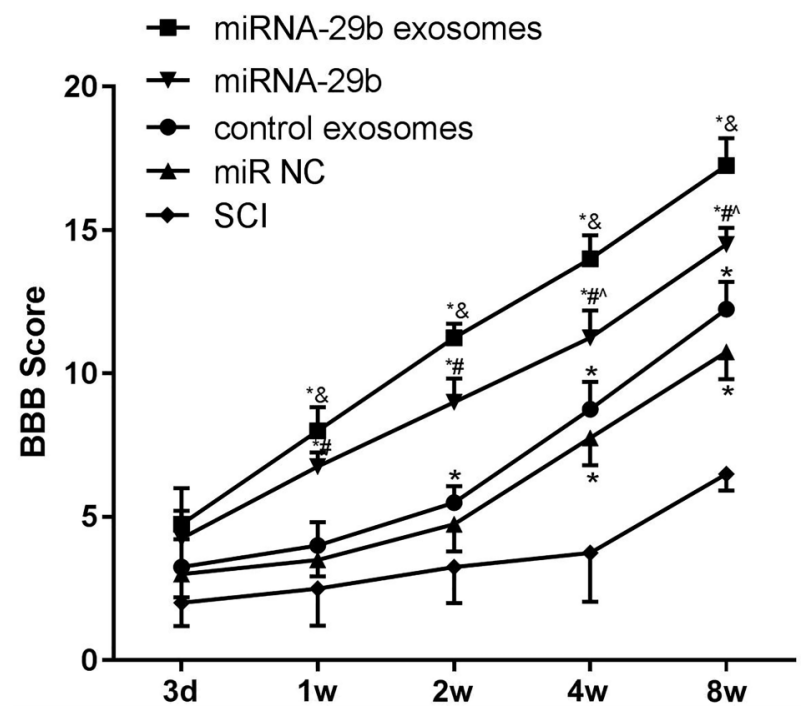

Figure 3. Basso Beattie Bresnahan (BBB) scores of rats at 3 days, 1, 2, 4, and 8 weeks post-injection. Data are reported as means $\pm \mathrm{SD}$. ${ }^{*} \mathrm{P}<0.05$ vs $\mathrm{SCl}$ group; ${ }^{\&} \mathrm{P}<0.05$ vs control exosomes; ${ }^{\# P}<0.05$ vs miR $\mathrm{NC}$; ${ }^{\wedge}<<0.05$ vs miRNA-29b exosomes (ANOVA)

exosomes group $(P<0.05)$. When compared with the miR NC group, a significantly lower number of contractile nerve cells was found in the miRNA-29b group $(P<0.05)$. Noteworthy, the miRNA-29b exosomes group exhibited a significantly lower number of contractile nerve cells than the miRNA-29b group $(P<0.05)$ (Figure 4).

\section{Injection of miRNA-29b exosomes promoted neuronal regeneration}

Neurofilament protein 200 (NF200), growth-associated protein-43 (GAP-43), and glial fibrillary acidic protein (GFAP) are three important proteins involved in neuronal regeneration. Immunohistochemical staining showed that relative low expression of NF200 and GAP-43 was observed in spinal cord tissues of $\mathrm{SCl}$ rats. The injection of BMSCs or exosomes significantly increased the expression of NF200 and GAP-43 in $\mathrm{SCl}$ rats at 2, 4, and 8 weeks post-injection $(P<0.05)$. In addition, the expression of NF200, GAP-43, and GFAP was significantly higher in the miRNA-29b exosomes group than in the control exosomes group $(\mathrm{P}<0.05)$, and also significantly higher in the miRNA-29b group than in the miR NC group at $1,2,4$, and 8 weeks post-injection $(P<0.05)$. Compared with the miRNA-29b group, the miRNA-29b exosomes group exhibited significantly higher NF200 and GAP-43 expression $(P<0.05)$ (Figure $5 A$ and $B$ ). In contrast, the expression changes of GFAP in spinal cord tissues of $\mathrm{SCl}$ rats in the 5 groups were just the opposite to NF200 at 1, 2, 4, and 8 weeks post-injection $(P<0.05)$ (Figure 5C).

\section{Discussion}

The pathophysiological changes of SCI mainly include two stages: primary injury and secondary injury (22). Primary injury involves spinal cord hemorrhage, nerve cell membrane rupture, as well as blood-brain barrier disruption caused by external traction or compression (23). Secondary injury is a series of chain reactions after primary injury, which involves local blood flow disorder, tissue ischemia and hypoxia, inflammatory cell infiltration, and nerve cell necrosis. Secondary injury results in further enlargement of the spinal cord injury area, more nerve cell death, and even nerve fiber deformation (24). In general, secondary injury is more severe and more difficult to manage than primary injury.

At present, many animal experiments and clinical studies have confirmed that BMSCs transplantation achieves a certain therapeutic effect on SCI $(25,26)$. Most studies believe that BMSCs play an important role on the repair of $\mathrm{SCl}$ by secreting various regulatory factors $(26,27)$. However, the clinical application of BMSCs transplantation is very limited due to low survival rate and differentiation rate in vivo (28). In 2010, Lai et al. (29) demonstrated for the first time that neurotrophic factors and nerve growth factors are exosomes secreted by MSCs. Exosomes are extracellular vesicles that contain a variety of biologically active substances, such as proteins, mRNAs, and miRNAs. Exosomes are able to promote intercellular communication through exchanging protein and genetic information (30). Teng et al. (31) confirmed that exosomes secreted by MSCs promote neovascularization, and inhibit inflammation in myocardial 


\section{$\mathrm{SCl}$}



$\operatorname{miR} N C$

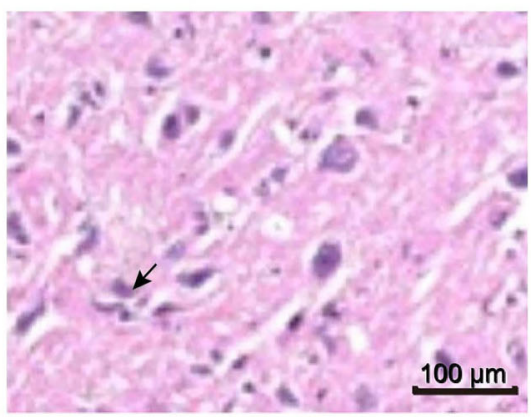

control exosomes



miRNA-29b

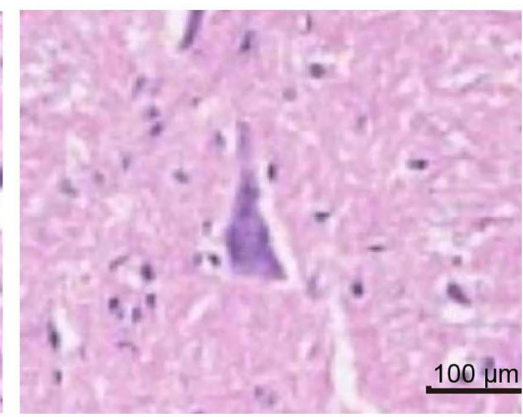

miRNA-29b exosomes
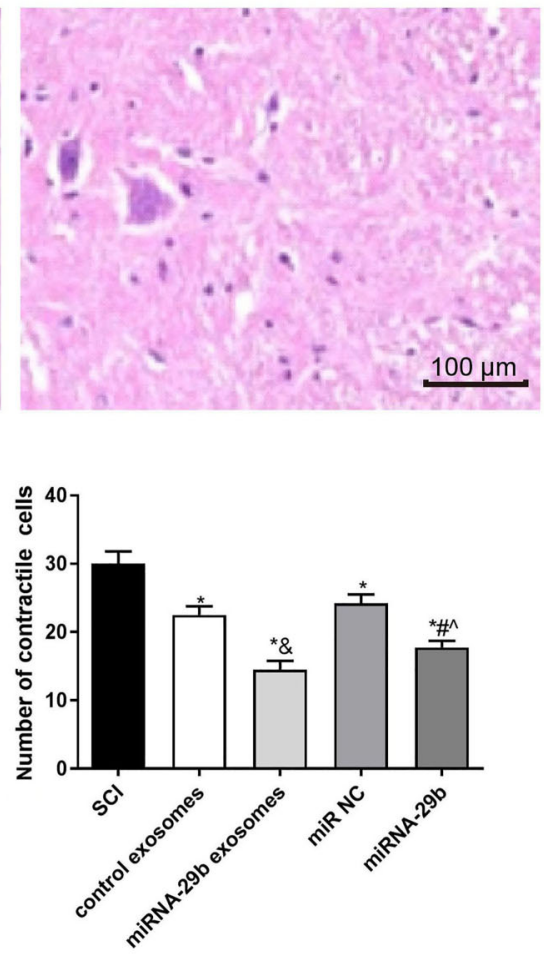

Figure 4. Contractile nerve cells were observed in spinal cord tissues of spinal cord injury (SCI) rats by HE staining at 4 weeks postinjection $(\times 400$, bar $=100 \mu \mathrm{m})$. Arrows indicate contractile nerve cells. Data are reported as means $\pm \mathrm{SD}$. ${ }^{*} \mathrm{P}<0.05 \mathrm{vs} \mathrm{SCl}$ group; ${ }^{\&} \mathrm{P}<0.05$ vs control exosomes; ${ }^{\#} \mathrm{P}<0.05$ vs miR NC; ${ }^{\wedge} \mathrm{P}<0.05$ vs miRNA-29b exosomes (ANOVA).

ischemic injury. Huang et al. (9) reported that exosomes secreted by MSCs promote functional recovery of SCI by relieving apoptosis and the inflammatory response, and stimulating angiogenesis. These findings indicate that the intervention of exosomes secreted by MSCs is a potential therapeutic strategy for $\mathrm{SCl}$. In this research, results were consistent with the above previous studies, and further illustrate that the injection of exosomes was effective in the treatment of $\mathrm{SCl}$.

miRNAs are a class of small non-coding RNAs, which exert important regulatory effects on cell biological behavior (32). Our results indicated that the injection of miRNA-29b exosomes was able to accelerate the repair of $\mathrm{SCl}$. In recent years, accumulated studies have shown that exosomes carrying miRNAs exert key repairing effects on damaged tissues. For example, Shen et al. (12) indicated that the brain injury in intracerebral hemorrhage rats is attenuated by the transplantation of exosomes carrying miR-133b. The mechanism involved in this process is that miR-133b can activate ERK1/2/CREB pathway, thereby inhibiting neuronal apoptosis and neurodegeneration. Chen et al. (15) showed that exosomes derived from miR-223 inhibitors-transfected BMSCs protect liver damage by regulating NLRP3 and caspase-1. Our findings were consistent with previous studies, and illustrated that the injection of miRNA-29b exosomes relieved the $\mathrm{SCl}$ in rats. Furthermore, we also found that the injection of miRNA29b exosomes significantly increased NF200 and GAP-43 positive neurons, and decreased GFAP positive neurons. NF200 is a skeletal structure of neural cells and axons (33). GAP-43 is a specific phosphoprotein on vertebrate nerve cell membrane that is considered a marker for synaptic plasticity, neuronal development, and regeneration (34). GFAP, a cytoskeletal protein, is the major component of glial cells (35). In the early stage of SCl, GFAP expression is upregulated, and some cytokines are secreted to promote axonal regeneration. However, in the late stage of $\mathrm{SCl}$, a large number of hyperplastic glial scars may inhibit the growth of axons (36). Since these three proteins can reflect the degree of neuronal regeneration in spinal cord tissues of $\mathrm{SCl}$ rats, our results further indicated that miRNA-29b exosomes can relieve $\mathrm{SCl}$ by regulating the expression of NF200, GAP-43, and GFAP.

In conclusion, the injection of exosomes secreted by miRNA-29b-modified BMSCs repaired $\mathrm{SCl}$ in rats. The relevant mechanism might be associated with the 


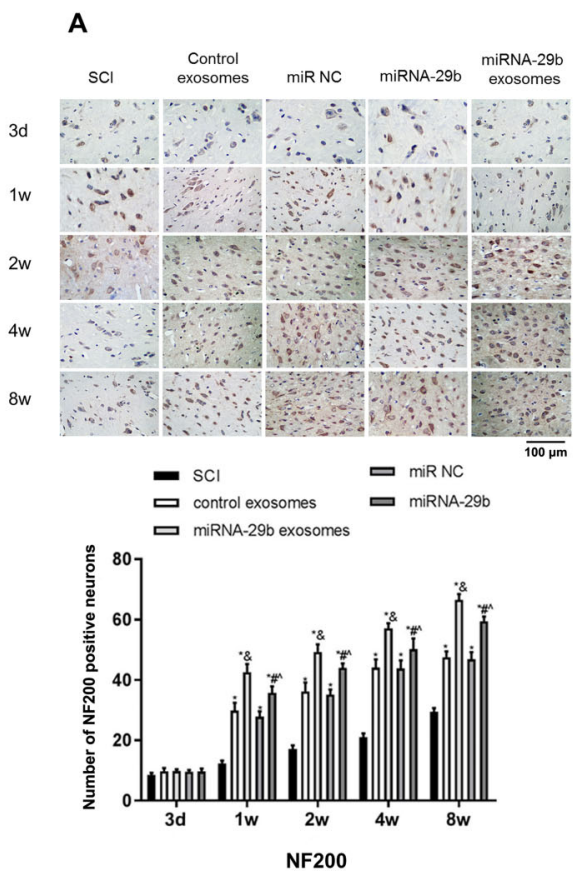

B


C


Figure 5. NF200 (A), GAP-43 (B), and GFAP (C) positive cells (brown) were observed in spinal cord tissues of spinal cord injury ( $\mathrm{SCl}$ ) rats by immunohistochemistry at 3 days, 1, 2, 4, and 8 weeks post-injection $(\times 500$, bar $=100 \mu \mathrm{m})$. Data are reported as means \pm SD. ${ }^{*} \mathrm{P}<0.05$ vs SCl group; ${ }^{\&} \mathrm{P}<0.05$ vs control exosomes; ${ }^{\#} \mathrm{P}<0.05$ vs miR $\mathrm{NC}$; ${ }^{\wedge} \mathrm{P}<0.05$ vs miRNA-29b exosomes (ANOVA).

regulation of proteins involved in neuronal regeneration, such as NF200, GAP-43, and GFAP. Exosomes secreted

\section{References}

1. Luo L, Albashari AA, Wang X, Jin L, Zhang Y, Zheng L, et al. Effects of transplanted heparin-poloxamer hydrogel combining dental pulp stem cells and bFGF on spinal cord injury repair. Stem Cells Int 2018; 2018: 2398521, doi: 10.1155/ 2018/2398521.

2. Orr MB, Simkin J, Bailey WM, Kadambi NH, Mcvicar AL, Veldhorst Ak, et al. Compression decreases anatomical and functional recovery and alters inflammation after contusive spinal cord injury. J Neurotrauma 2017; 34: 2342-2352, doi: 10.1089/neu.2016.4915.

3. Papastefanaki F, Matsas R. From demyelination to remyelination: The road toward therapies for spinal cord injury. Glia 2015; 63: 1101-1125, doi: 10.1002/glia.22809.

4. Karaoz E, Kabatas S, Duruksu G, Okcu A, Subasi C, Ay B, et al. Reduction of lesion in injured rat spinal cord and partial functional recovery of motility after bone marrow derived mesenchymal stem cell transplantation. Turk Neurosurg 2012; 22: 207-217, doi: 10.5137/1019-5149.JTN.5412-11.1.

5. Luo F, Liu T, Wang J, Li J, Ma P, Ding $\mathrm{H}$, et al. Bone marrow mesenchymal stem cells participate in prostate carcinogenesis and promote growth of prostate cancer by cell fusion in vivo. Oncotarget 2016; 7: 30924-30934, doi: 10.18632/ oncotarget.9045. by miRNA-29b-modified BMSCs may be a promising therapeutic agent for $\mathrm{SCl}$ in clinical practice.

6. Yang Y, Song HL, Zhang W, Wu BJ, Fu NN, Dong C, et al. Heme oxygenase-1-transduced bone marrow mesenchymal stem cells in reducing acute rejection and improving small bowel transplantation outcomes in rats. Stem Cell Res Ther 2016; 7: 164, doi: 10.1186/s13287-016-0427-8.

7. Wang X, Botchway BOA, Zhang Y, Yuan J, Liu X. Combinational treatment of bioscaffolds and extracellular vesicles in spinal cord injury. Front Mol Neurosci 2019; 12: 81, doi: 10.3389/fnmol.2019.00081.

8. Ludwig AK, Giebel B. Exosomes: small vesicles participating in intercellular communication. Int $\mathrm{J}$ Biochem Cell Biol 2012; 44: 11-15, doi: 10.1016/j.biocel.2011.10.005.

9. Huang JH, Yin XM, Xu Y, Xu CC, Lin X, Ye FB, et al. Systemic administration of exosomes released from mesenchymal stromal cells attenuates apoptosis, inflammation and promotes angiogenesis after contusion spinal cord injury in rats. J Neurotrauma 2017; 34: 3388-3396, doi: 10.1089/neu.2017.5063.

10. Sun G, Li G, Li D, Huang W, Zhang R, Zhang $H$, et al. hucMSC derived exosomes promote functional recovery in spinal cord injury mice via attenuating inflammation. Mater Sci Eng C Mater Biol Appl 2018; 89: 194-204, doi: 10.1016/ j.msec.2018.04.006. 
11. Wang L, Pei S, Han L, Guo B, Li Y, Duan R, et al. Mesenchymal stem cell-derived exosomes reduce a1 astrocytes via downregulation of phosphorylated NFKB p65 subunit in spinal cord injury. Cell Physiol Biochem 2018; 50: 1535-1559, doi: 10.1159/000494652.

12. Shen H, Yao X, Li H, Li X, Zhang T, Sun Q, et al. Role of exosomes derived from mir-133B modified mscs in an experimental rat model of intracerebral hemorrhage. $\mathrm{J} \mathrm{Mol}$ Neurosci 2018; 64: 421-430, doi: 10.1007/s12031-0181041-2.

13. Li S, Zhang R, Yuan Y, Yi S, Chen Q, Gong L, et al. Mir-340 regulates fibrinolysis and axon regrowth following sciatic nerve injury. Mol Neurobiology 2017; 54: 4379-4389, doi: 10.1007/s12035-016-9965-4.

14. Wang N, Yang W, Xiao T, Miao Z, Luo W, You Z, et al. Possible role of miR-204 in optic nerve injury through the regulation of GAP-43. Mol Med Rep 2018; 17: 3891-3897, doi: 10.3892/mmr.2017.8341.

15. Chen L, Lu FB, Chen DZ, Wu JL, Hu ED, Xu LM, et al. BMSCs-derived miR-223-containing exosomes contribute to liver protection in experimental autoimmune hepatitis. Mol Immunol 2018; 93: 38-46, doi: 10.1016/j.molimm.2017. 11.008.

16. Zhang $\mathrm{Y}$, Wu L, Wang $\mathrm{Y}$, Zhang $\mathrm{M}$, Li L, Zhu D, et al. Protective role of estrogen-induced miRNA-29 expression in carbon tetrachloride-induced mouse liver injury. J Biol Chem 2012; 287: 14851-14862, doi: 10.1074/jbc.M111.314922.

17. Ye Y, Hu Z, Lin Y, Zhang C, Perez-Polo JR. Downregulation of microRNA-29 by antisense inhibitors and a PPAR- $\gamma$ agonist protects against myocardial ischaemia-reperfusion injury. Cardiovasc Res 2010; 87: 535-544, doi: 10.1093/cvr/ cvq053.

18. Galimov A, Merry TL, Luca E, Rushing EJ, Mizbani A, Turcekova K, et al. microRNA-29a in adult muscle stem cells controls skeletal muscle regeneration during injury and exercise downstream of fibroblast growth factor-2. Stem Cells 2016; 34: 768-780, doi: 10.1002/stem.2281.

19. Eisenreich A, Langer S, Herlan L, Kreutz R. Regulation of podoplanin expression by microRNA-29b associates with its antiapoptotic effect in angiotensin II-induced injury of human podocytes. J Hypertension 2016; 34: 323-331, doi: 10.1097/ HJH.0000000000000799.

20. Basso DM, Beattie MS, Bresnahan JC. A sensitive and reliable locomotor rating scale for open field testing in rats. J Neurotrauma 1995; 12: 1-21, doi: 10.1089/neu.1995.12.1.

21. Song JL, Zheng W, Chen W, Qian Y, Ouyang YM, Fan CY. Lentivirus-mediated microRNA-124 gene-modified bone marrow mesenchymal stem cell transplantation promotes the repair of spinal cord injury in rats. Exp Mol Med 2017; 49: e332, doi: 10.1038/emm.2017.48.

22. Garcia E, Aguilar-Cevallos J, Silva-Garcia R, Ibarra A. Cytokine and growth factor activation in vivo and in vitro after spinal cord injury. Mediators Inflamm 2016; 2016: 9476020, doi: 10.1155/2016/9476020.

23. Kumar H, Ropper AE, Lee SH, Han I. Propitious therapeutic modulators to prevent blood-spinal cord barrier disruption in spinal cord injury. Mol Neurobiol 2017; 54: 3578-3590, doi: 10.1007/s12035-016-9910-6.
24. White-Schenk D, Shi R, Leary JF. Nanomedicine strategies for treatment of secondary spinal cord injury. Int $J$ Nanomedicine 2015; 10: 923-938, doi: 10.2147/IJN.S75686.

25. Lin $L$, Lin $H$, Bai $S$, Zheng $L$, Zhang $X$. Bone marrow mesenchymal stem cells (BMSCs) improved functional recovery of spinal cord injury partly by promoting axonal regeneration. Neurochem Int 2018; 115: 80-84, doi: 10.1016/j.neuint.2018.02.007.

26. Zhao H, Cheng L, Du X, Hou Y, Liu Y, Cui Z, et al. Transplantation of cerebral dopamine neurotrophic factor transducted BMSCs in contusion spinal cord injury of rats: promotion of nerve regeneration by alleviating neuroinflammation. Mol Neurobiol 2016; 53: 187-199, doi: 10.1007/ s12035-014-9000-6.

27. Ritfeld GJ, Patel A, Chou A, Novosat TL, Castillo DG, et al. The role of brain-derived neurotrophic factor in bone marrow stromal cell-mediated spinal cord repair. Cell Transplant 2015; 24: 2209-2220, doi: 10.3727/096368915X686201.

28. Tan $Y$, Uchida K, Nakajima $H$, Guerrero AR, Watanabe $S$, Hirai T, et al. Blockade of interleukin 6 signaling improves the survival rate of transplanted bone marrow stromal cells and increases locomotor function in mice with spinal cord injury. J Neuropathol Exp Neurol 2013; 72: 980-983, doi: 10.1097/NEN.0b013e3182a79de9.

29. Lai RC, Arslan F, Lee MM, Sze NSK, Choo A, Chen TS, et al. Exosome secreted by MSC reduces myocardial ischemia/reperfusion injury. Stem Cell Res 2010; 4: 214-222, doi: 10.1016/j.scr.2009.12.003.

30. Salimian J, Mirzaei H, Moridikia A, Harchegani AB, Sahebkar A, Salehi H. Chronic obstructive pulmonary disease: MicroRNAs and exosomes as new diagnostic and therapeutic biomarkers. J Res Med Sci 2018; 23: 27, doi: 10.4103/jrms.JRMS_1054_17.

31. Teng X, Chen L, Chen W, Yang J, Yang Z, Shen Z. Mesenchymal stem cell-derived exosomes improve the microenvironment of infarcted myocardium contributing to angiogenesis and anti-inflammation. Cell Physiol Biochem 2015; 37: 2415-2424, doi: 10.1159/000438594.

32. Xu M, Chen X, Huang $Z$, Chen $D, Y u$ B, Chen $H$, et al. MicroRNA-139-5p suppresses myosin heavy chain I and Ila expression via inhibition of the calcineurin/NFAT signaling pathway. Biochem Biophys Res Commun 2018; 500: 930-936, doi: 10.1016/j.bbrc.2018.04.202.

33. Lee MK, Xu Z, Wong PC, Cleveland DW. Neurofilaments are obligate heteropolymers in vivo. J Cell Biol 1993; 122: 1337-1350, doi: 10.1083/jcb.122.6.1337.

34. Benowitz LI, Routtenberg A. GAP-43: an intrinsic determinant of neuronal development and plasticity. Trends Neurosci 1997; 20: 84-91, doi: 10.1016/S0166-2236(96) 10072-2.

35. Cheng H, Wu JP, Tzeng SF. Neuroprotection of glial cell line-derived neurotrophic factor in damaged spinal cords following contusive injury. J Neurosci Res 2002; 69: 397-405, doi: 10.1002/jnr.10303.

36. Song HM, Luo Y, Li DF, Wei CK, Hua KY, Song JL, et al. MicroRNA-96 plays an oncogenic role by targeting FOXO1 and regulating AKT/FOXO1/Bim pathway in papillary thyroid carcinoma cells. Int J Clin Exp Pathol 2015; 8: 9889-9900. 